\title{
DEUTSCHES ELEKTRONENSYNCHROTRON
}

DESY $75 / 35$

September 1975
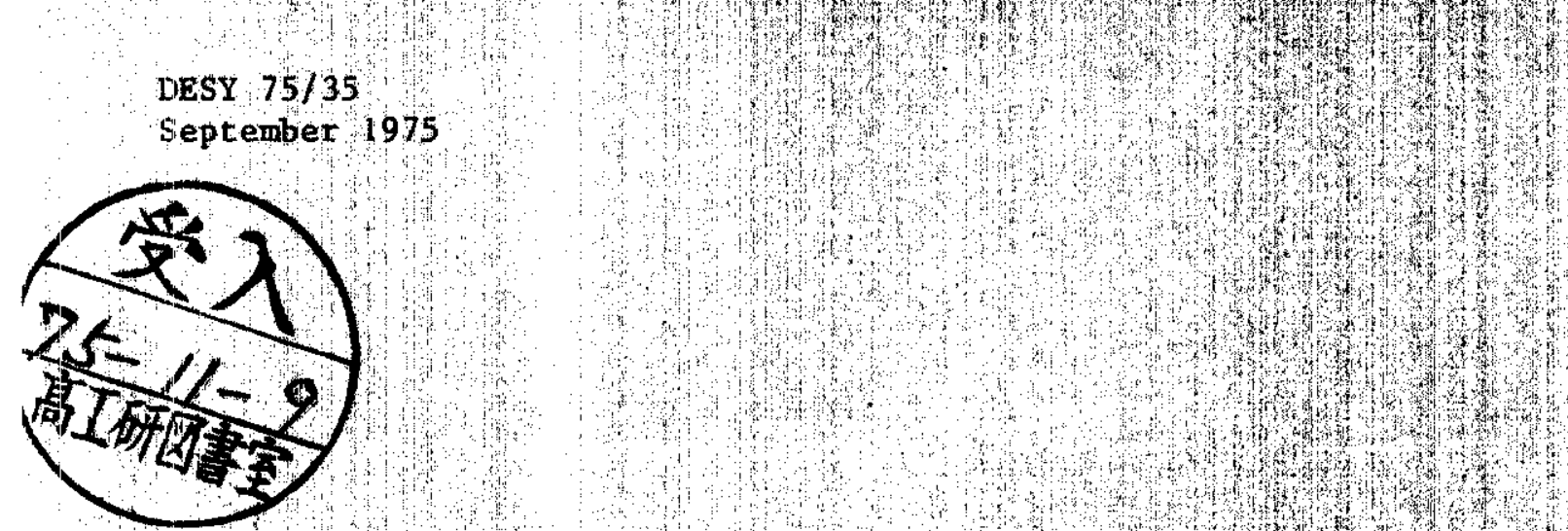

Jets in Inclusive eté and Leproptodwotos

by

M. Gronau, $\mathbf{T}, \mathrm{F}$, Walsh and $\mathrm{T}$, Zarm

Deutsches tilektronen-synahrotron DEST, Hombuth

\section{W. S. Lam}

Theoretical Bhysucs, University of Bieterdza.
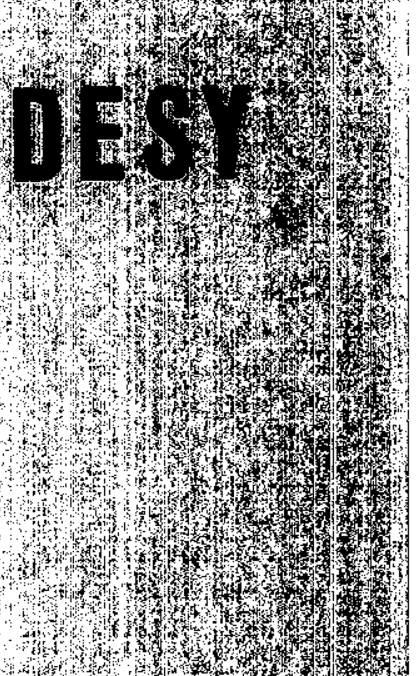

$$
\begin{aligned}
& \text { 147 }
\end{aligned}
$$
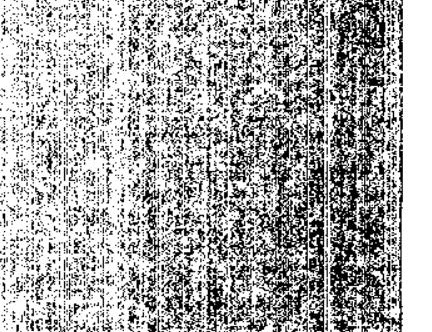

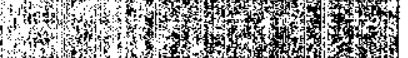

$$
\begin{aligned}
& \text { 4.73 } \\
& \text { 1.7, } \\
& \text { 137x }
\end{aligned}
$$$$
\text { 3.7. W. }
$$$$
\text { 1. }
$$$$
\text { P. }
$$$$
\text { H. }
$$$$
\text { 115 }
$$$$
\text { H.t. }
$$

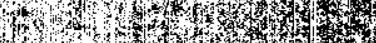$$
\text { mathes }
$$

2 HAMBURG52 W NOTASTECI
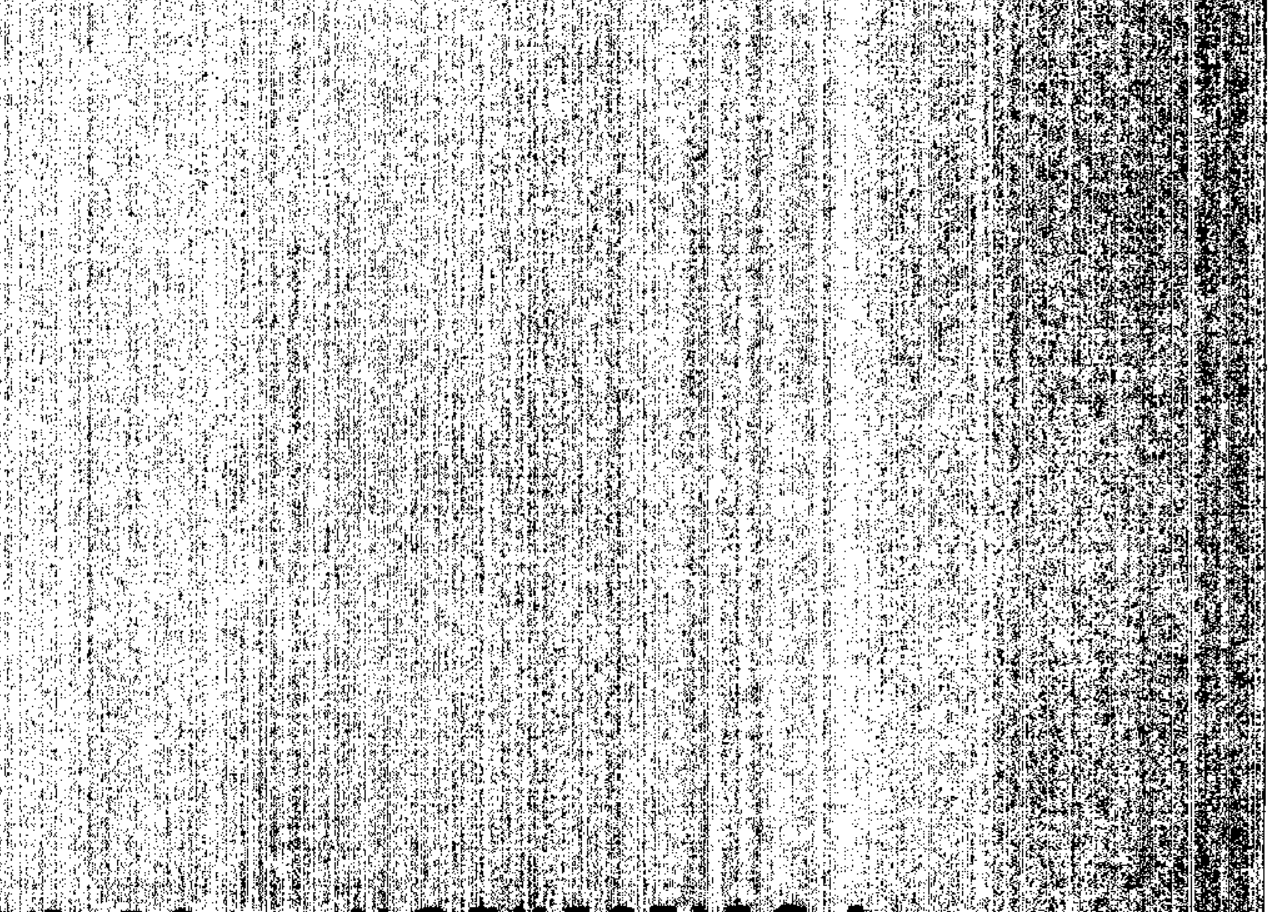

H3

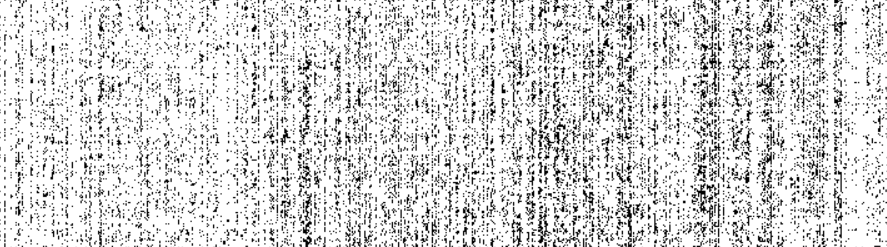

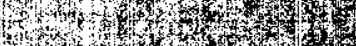

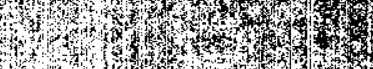
Wh+m 
To be sure that your preprints are promptly included in the HIGH ENERGY PHYSICS INDEX.

send them to the following address (if possible by air mail) :

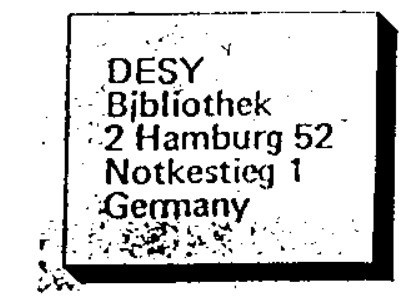




\title{
Jets In Inclusive $\mathrm{e}^{+} \mathrm{e}^{-}$And Leptoproduction
}

\author{
M. Gronau $^{+}$, T.F. Walsh and Y. Zarmi ${ }^{++}$ \\ Deutsches Elektronen-Synchrotron DESY, Hamburg, Germany \\ and \\ W.S. Lam \\ Theoretical Physics, University of Bielefeld
}

\section{Abstract}

The assumption of jets with bounded $p_{T}$ leads to the following consequences:

a) In $e^{+} e^{-}$annihilation the coefficient of the $\cos ^{2} \theta$ term $(\alpha)$ of single particle inclusive distributions exhibits a unique dependence on the observed particle momentum. This dependence provides a measure of $\left\langle p_{T}^{2}\right\rangle$ in a jet.

b) In two particle inclusive annihilation the average of the squared relative $P_{T}$ is determined by the same parameter and by the momenta of the two particles.

c) The observation that in the current fragmentation region of leptoproduction the $p_{T}$ distribution of fast hadrons is broader than that of slow ones is explained.

$+\quad$ On leave of absence from the Physics Department, Technion, Israel Institute of Technology, Haifa.

++ On leave of absence from the Physics Department, The Weizmann Institute, Rehovot. 


\section{Introduction}

A basic ingredient of the parton model is the presence of a transverse momentum cut-off ${ }^{1)}$. This cut-off is assumed to apply to the distribution of partons in a hadron, and to the distribution of hadrons "radiated" by a parton. It is essential to the explanation of scaling phenomena.

Tests of the cut-off itself are scarce. A sharp cut-off leads to the prediction that in deep inelastic processes with spin $1 / 2$ partons, $\sigma_{L} / \sigma_{T}$ behaves as $1 / q^{2}$ (for fixed $\omega=-2 p \cdot q / q^{2}$ ), and to the vanishing (for large $q^{2}$ ) of azimuthal asymmetries of hadrons produced in leptoproduction ${ }^{2}$. It also predicts bounded $\left\langle p_{T}^{2}\right\rangle$ for the produced hadrons around the current-target axis. And, of course, the cut-off leads to the prediction of jets in $e^{+} e^{-}$ annihilation. 3)

We discuss here some straightforward consequences of the existence of a transverse momentum cut-off. In $e^{+} e^{-}$annihilation into one hadron plus anything the hadron angular distribution becomes, as is well known, $1+\cos ^{2} \theta$ for 1 arge momentum hadrons (assuming spin $1 / 2$ partons). At low momenta the distribution should be isotropic. We show that the transition between the two extremes is controlled by the mean squared transverse momentum of hadrons with respect to the jet axis. The same is true of deviations from scaling at intermediate momenta. In two particle inclusive $e^{+} e^{-}$annihilation the relative $\left\langle p_{T}^{2}\right\rangle$ of hadrons coming out in opposite hemispheres is also related to $\left\langle p_{T}^{2}\right\rangle$ of hadrons relative to a jet axis. Similarly, the transverse momenta of hadrons produced in the forward region of current + hadron $\rightarrow$ hadron $+X$ are related to the $\left\langle P_{T}^{2}\right\rangle$ of $e^{+} e^{-}$annihilation. As a consequence, we find a broadening of the 
distribution of current fragments as $x_{f}$ increases towards 1 . All these features may serve as tests of the cut-off assumption originally introduced in order to obtain Bjorken scaling.

\section{Consequences of the cut-off}

The parton model predicts a jet structure for hadronic $e^{+} e^{-}$annihilation events. Searches for jets at low energies $\left({\sqrt{Q^{2}}}^{2} 4.8 \mathrm{GeV}\right){ }^{4}$ ) have not been conclusive. Higher energy data $\left(\sqrt{Q^{2}}=6.2\right.$ and $\left.7.4 \mathrm{GeV}\right)$ 5) indicate jet structure, consistent with a parton model involving spin $1 / 2$ constituents. In the following we assume a jet structure and explore the (mostly kinematical) consequences for $\mathrm{e}^{+} \mathrm{e}^{-}$annihilation and inclusive leptoproduction. Caveats are collected at the end of the section in order to avoid mutilating the otherwise simple discussion.

\section{2a. Single Particle Inclusive $e^{+} e^{-}$Annihilation}

In a jet the distribution of hadron momenta perpendicular to the axis is sharply cut of $f$. In the parton model this axis is defined by the original parton anti-parton direction. Large momentum hadrons should closely follow the jet axis and exhibit its angular distribution. On the other hand, low momentum hadrons do not know which way the parton went (ox know nothing about partons) and should be distributed isotropically. 6)

Assuming annihilation through a single photon the jet axis angular distribution

$$
\begin{aligned}
& \text { is }\left(\alpha_{j}=\alpha_{j e t}\left(Q^{2}\right)\right) \\
& \frac{d \sigma}{d \Omega_{j e t}} \alpha 1+\alpha_{j} \cos ^{2} \theta_{j}+\alpha_{j} P^{2} \sin ^{2} \theta_{j} \cos 2 \varphi_{j}
\end{aligned}
$$


$-3-$

$P$ is the $\mathrm{e}^{-}$or $\mathrm{e}^{+}$polarization perpendicular to the $\mathrm{e}^{+} \mathrm{e}^{-}$orbit $\mathrm{plane}$;

$\theta_{j}$ and $\varphi_{j}$ are the polar and azimuthal angles of the jet relative to the $\mathrm{e}^{+} \mathrm{e}^{-}$axis and the plane of the $\mathrm{e}^{+} \mathrm{e}^{-}$ring (we assume zero crossing angle between the beams). In general, $-1 \leq \alpha_{j} \leq 1$, with $\alpha_{j}=1-O\left(1 / Q^{2}\right)(-1)$ for spin $1 / 2$ (0) partons.

A prior, the single particle inclusive distribution relative to the jet axis may depend on $Q^{2}, x=2 p / \sqrt{Q^{2}}$ and $p_{T}=p \sin \tilde{\theta}$. Denote it by $f\left(Q^{2}, x, p_{T}\right)$ (In the parton model this is the fragmentation function $\left.D_{q}^{h}\left(x, p_{T}\right)\right)^{1)}$. Here $\tilde{\theta}$ is the angle between the particle momentum $p$ and the jet axis. Consequently,

$$
Q^{2} \frac{d \sigma}{d^{3} p / E} \propto \int \frac{d \sigma(j e t)}{d \Omega_{j e t}} \cdot f\left(Q^{2}, x, p_{T}\right) d \Omega_{j e t}
$$

With the help of Fig. 1 we find

$$
\begin{aligned}
Q^{2} \frac{d \sigma}{d^{3} P / E} \alpha\left(I_{1}+\frac{1}{2} \alpha_{j} I_{2}\right)\left[1+\alpha\left(Q^{2}, P\right) \cos ^{2} \theta\right. & \left.+\alpha\left(Q^{2}, P\right) P^{2} \sin ^{2} \theta \cos 2 \varphi\right] \\
& +\alpha_{j} \frac{1-3 / 2 \cdot I_{2} / I_{1}}{1+1 / 2 \cdot \alpha_{j} I_{2} / I_{1}}
\end{aligned}
$$

where

$$
\left.\begin{array}{l}
I_{1}=\int d \cos \tilde{\theta} f\left(Q^{2}, x, p_{T}\right)=\frac{1}{2 p^{2}} \int \frac{d p_{T}^{2}}{\sqrt{1-p_{T}^{2} / p^{2}}} f\left(Q^{2}, x, p_{T}\right) \\
I_{2}=\int d \cos \tilde{\theta} \sin ^{2} \tilde{\theta} f\left(Q^{2}, x, p_{T}\right)= \\
\frac{1}{2 p^{2}} \int \frac{d p_{T}^{2}}{\sqrt{1-p_{T}^{2} / p^{2}}} \cdot \frac{p_{T}^{2}}{p^{2}} f\left(Q^{2}, x, p_{T}\right)
\end{array}\right\}
$$


In general, $\alpha$ is a function of the particle momentum $p$. For large $\mathrm{p}$,

$$
\begin{aligned}
& I_{2} / I_{1}=\frac{\left\langle p_{T}^{2}\right\rangle}{p^{2}}+O\left(\left(\frac{\left\langle p_{T}^{2}\right\rangle}{p^{2}}\right)^{2}\right) \\
& \left\langle p_{T}^{2}\right\rangle=\frac{\int p_{T}^{2} f\left(Q^{2}, x_{,} p_{T}\right) d p_{T}^{2}}{\int f\left(Q^{2}, x, P_{T}\right) d p_{T}^{2}}
\end{aligned}
$$

independent of the detailed form of $f$. Then,

$$
\alpha \cong \alpha_{j}\left(1-\frac{\alpha_{j}+3}{2} \frac{\left\langle p_{T}^{2}\right\rangle}{p^{2}}\right)
$$

$\left\langle p_{T}^{2}\right\rangle$ may depend on $Q^{2}$ and $x$ through the function $f\left(Q^{2}, x, p_{T}\right\rangle$. This dependence is probably weak. If $f$ factorizes into a function of $Q^{2}, x$ times a function of $p_{T}$ there is no $Q^{2}$ and $x$ dependence in $\left\langle p_{T}^{2}\right\rangle$. From now on we ignore such a dependence.

In the high energy limit, with all spin $1 / 2$ partons, $\alpha_{j} \rightarrow 1$. We therefore predict that asymptotically

$$
\alpha \cong 1-2\left\langle p_{T}^{2}\right\rangle / p^{2}
$$

is a function of $p$ alone. Once the existence of $j$ ets and the momentum dependence of $\alpha$ are established, eq. (7) permits a model independent determination of $\left\langle p_{T}^{2}\right\rangle$, the mean squared transverse momentum of hadrons relative to the jet axis. 
Asymptotically, the parton model predicts a constant $R=\sigma_{\text {nad }} / C_{\mu} \mu$, $Q^{2} \frac{d \sigma}{d x} \quad$ a function of $x$ alone, and $\quad \alpha=1$ (or $\sigma_{L} / \sigma_{T}=\frac{1-\alpha}{1+\alpha}=0$ ). The last two properties are related. Let us perform the angle integration in eq. (3), set $f\left(G^{2}, x, p_{T}\right)=f(x) \cdot g\left(p_{T}\right) \quad$ and expand the result in powers of $p^{-2}$. We find for $\alpha_{j}=1$ and $\left.p^{2}\right\rangle\left\langle f_{T}^{2}\right\rangle$ :

$$
\begin{aligned}
Q^{2} \times \frac{d \sigma}{d x} \propto & \times f(x)\left(1+\frac{1}{2} \frac{\left\langle p_{T}^{2}\right\rangle}{p^{2}}+\cdots\right) \\
& =x f(x)\left(1+\frac{1}{4}(1-\alpha)+\cdots\right)
\end{aligned}
$$

Thus, $\alpha \neq 1$ in some $x$ range implies that scaling must be violated there

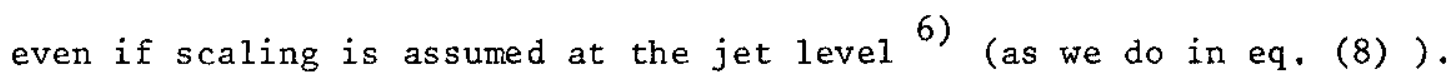

We have remarked that $\alpha(p)$ is independent of the details of $f\left(Q^{2}, x, p_{\tau}\right)$ for large $p$. This is no longer true of eq. (8). Suppose that the correct scaling variable at the jet level is not $\mathrm{x}$ but rather the commonly used $x_{11}=2 p_{11} / \sqrt{C^{2}}=x \sin \tilde{\theta} \quad$. Denote the parton fragmentation function by $\tilde{f}\left(x_{11}, p_{T}\right)$. For large $p, \alpha(p)$ coincides with eqs. (6-7) to $\boldsymbol{O}\left(1 / p_{4}\right)$. However, eq. (8) is replaced by

$$
Q^{2} \times \frac{d \sigma}{d x} \propto x \tilde{f}(x)\left(1+\frac{1}{2} \frac{\left\langle p^{2}\right\rangle}{p^{2}}-\frac{1}{2} \frac{x \tilde{f}(x)}{\tilde{f}(x)} \cdot \frac{\left\langle p^{2} \frac{T}{p^{2}} \geq\right.}{p^{2}}+\cdots\right)
$$

Note that as long as $\tilde{f}(x)$ is a decreasing function of $x$, the scaling limit is approached from above in either case. The deviations from scaling depend, however, on the choice of scaling variable at the jet level. The choice of $x_{\|}$as the scaling variable for the fragmentation function clearly 
leads to difficulties as $\mathrm{x} \rightarrow 1$, since there the last term in eq. (9)

behaves as $(1-x)^{-1}$ (for $\left.\tilde{f}(x) \propto(1-x)^{n}\right)$. This indicates that $x_{1}$ may be the wrong variable, at least for large $x$. Choice of $\mathbf{X}_{11}$ does not reflect the fact that for large $x$ one remains at a fixed distance from the kinematical boundary as $\tilde{\theta}$ varies. When $\widetilde{\theta}$ increases, so does $p_{T}$. This tends to push $\tilde{f}\left(x_{l l}, p_{T}\right)$ downwards. However, at the same time, since the observed fixed quantity is $x, x_{11}=x \cos \tilde{\theta}$ decreases, which tends to push $\tilde{f}\left(x_{n}, p_{T}\right)$ upwards. It is this which leads to the large scaling violation for $x$ near 1 in eq. (9). Obviously the correct choice of variable is a dynamical question which we cannot answer here.

Until now we have ignored the effect on $\left\langle p_{T}^{2}\right\rangle$ and approach to scaling due to the presence of different types of particles. For example, an increasing Kaon fraction at large $x$ would affect $\left\langle p_{T}^{2}\right\rangle$. However, one can in principle establish $\left\langle p_{T}^{2}\right\rangle$ for each species as we have described, and we regard this as a detail. Finally, we have ignored the effects arising from the existence of different parton species. As long as $\alpha_{j}$ is the same for all species, none of our conclusions are affected. In this case $\left\langle p_{T}^{2}\right\rangle$ also include an average over several parton fragmentation functions (not just one $f\left(x, p_{T}\right)$ ), weighted by parton charges. For large $p$ we still have $\alpha(p) \rightarrow \alpha_{j}$. If, on the other hand, we include partons with different spins, things change. For example, with spin $0,1 / 2$ partons one has

$$
\alpha_{j}=\frac{\sum Q_{i}\left(\frac{1}{2}\right)^{2}-\frac{1}{2} \sum Q_{i}(0)^{2}}{\sum Q_{i}\left(\frac{1}{2}\right)^{2}+\frac{1}{2} \sum Q_{i}(0)^{2}}
$$


Since $\alpha(p)$ for a particle is an average over different fragmentation functions, it no longer approaches $\boldsymbol{\alpha}_{j}$ as $p$ becomes large (except. for the improbable case that the fragmentation of partons into hadrons does not depend on the parton spin).

If some of the partons have larger masses than others, this also affects our conclusions. For example, if the observed increase in $R$ around $\sqrt{Q^{2}} \cong 4 \mathrm{GeV}$ is due to a threshold associated with new parton types, then $\alpha(p)$ could be a different function of $p$ below and well above this threshold. The approach to scaling of $Q^{2} \frac{d \sigma}{d x}$ is also affected. The details depend on the fragmentation functions of the new heavy partons into stable hadrons. In the transition region one expects two contributions to $\alpha$. One (light partons) has $\alpha_{j} \cong 1$ and the other (heavy new partons) has $\alpha_{j}^{\text {new }} \cong\left(1-m_{\varphi^{2}}^{2}\right) /\left(1+m_{p}^{2} / Q^{2}\right)$. The latter is small near the new particle threshold $\left(Q^{2} \sim 4 m_{p}^{2}\right)$. Even for this reason alone large breaking of scaling is expected near new thresholds.

2b. Two Particle Distributions in $\mathrm{e}^{+} \mathrm{e}^{-}$

We now consider $p_{T}$ correlations in $e^{+} e^{-} \rightarrow h_{1}+h_{2}+x$, with $h_{1}$ and $h_{2}$ in opposite hemispheres (relative angle close to $180^{\circ}$ ) and $x_{1}, x_{2}$ not too small $\left(x_{i}=2 p_{i} / \sqrt{Q^{2}}\right)$. We may then assume that they come from two opposite jets, and are emitted independently. Each of them has a mean $\left\langle p_{T}^{2}\right\rangle$ with respect to its jet $\left(\left\langle p_{T}^{2}\right\rangle_{1 / j}\right.$ and $\left.\left\langle p_{T}^{2}\right\rangle_{2 / j}\right)$ and a mean relative squared transverse momentum $\left\langle p_{T}^{2}\right\rangle_{2 / 1}$. The latter can be measured. With the aid of fig. (2) we find

$$
\left\langle p_{T}^{2}\right\rangle_{2 / 1}=\left\langle p_{T}^{2}\right\rangle_{2 / j}+\frac{p_{2}^{2}}{p_{1}^{2}}\left\langle p_{T}^{2}\right\rangle_{1 / j}-\frac{3}{2} \frac{\left\langle p_{T}^{2}\right\rangle_{1 / j}\left\langle p_{T}^{2}\right\rangle_{2 / j}}{p_{1}^{2}}
$$


For finite $x_{1}, x_{2}$, under the cut-off assumption we can ignore the last term in eq. (11). If the two observed particles are charge conjugates $\left(\pi^{+} \bar{I}^{-}, K^{+} K^{-}, p, \bar{p} \quad\right.$ etc. $)$ we have after averaging over parton types

$$
\left\langle p_{T}^{2}\right\rangle_{2 / 1}=\left\langle p_{T}^{2}\right\rangle\left(1+\frac{x_{2}^{2}}{x_{1}^{2}}\right)
$$

with the same $\left\langle\boldsymbol{p}_{T}^{2}\right\rangle$ as for the single particle case. Eq. (12) is valid only for $\left.p_{i}^{2}\right\rangle\left\langle\left\langle p_{T}^{2}\right\rangle_{i / j e t}(i=1,2)\right.$. It can be used without having to experimentally define a jet axis. It also indicates that in order to use particle no. 1 for the approximate definition of a jet axis, $x_{1}$ must be close to $1\left(x_{1}\right\rangle .5$, say). Otherwise $\left\langle p_{T}^{2}\right\rangle_{2 / 1}$ may not be as small as $\left\langle p_{T}^{2}\right\rangle_{2 / j}$.

An immediate consequence of the assumed sharp cut-off in transverse momenta of hadrons relative to a jet axis is the absence of azimuthal asymetry in $\left.e^{+} e^{-} \rightarrow h_{1}+h_{2}+x \quad 7\right)$. In principle using $p_{1}$ as an axis, the cross section could depend on the azimuthal angle defined by $p_{2}$ around this axis. Such a dependence is predicted to disappear when $Q^{2}$ increases (for fixed $x_{1}, x_{2}$ ). The proof is essentially the same as in inclusive electroproduction 2 ). This prediction is a genuine test of the $p_{T}$-cut-off assumption with spin $1 / 2$ partons, since the more common prediction of $\sigma_{L} / \sigma_{T} \rightarrow 0$ can be easily obtained in other models.

\section{2c. The Parton Fragmentation Region in Leptoproduction}

Consider now the $P_{T}$ distribution of hadrons in the parton fragmentation region of electro- or neutrino-production. It is customary to view this process in the Breit frame, with the current-target direction as z-axis. 
After absorbing the current, the target parton has its $P_{\| 1}$ with respect to this axis reversed, while its $p_{T}$ is unaffected. The final hadron distribution is assumed to be of the form ${ }^{1)}$

$$
f\left(p_{1}, p_{T}\right) \sim d^{q}\left(x, p_{T}(q / N)\right) \cdot D_{q}^{h}\left(z, p_{T}(h / q)\right)
$$

which must be summed over (quarks) partons and integrated over internal variables. Here $x=-q^{2} / 2 p_{N} \cdot q$ and $z=2 p h \cdot q / q^{2}$. Averaging again over quark types and applying the same arguments as before (see Fig. 3) we find (neglecting $O\left(\frac{1}{q^{2}}\right)$ terms)

$$
\left\langle p_{T}^{2}\right\rangle_{h /(\gamma-N)} \cong\left\langle p_{T}^{2}\right\rangle_{h / q}+\xi^{2}\left\langle p_{T}^{2}\right\rangle_{q / N}
$$

This predicts a broadening of the $P_{T}$ distribution for large $z$. Practically, when $z$ is not close to $0\left(z>\sim \cdot 2\right.$, say) and $q^{2}$ is not to small $\left(\sqrt{-q^{2}}\right.$ larger than mass of observed particle) one may replace $z$ by the usual Feynman scaling variable $x_{F}$. However due to arguments similar to those presented in section $2 a$. (in discussing $x$ versus $x_{11}$ ) replacing $z$ by $x_{F}$ will have an effect on $\left\langle P_{T}^{2}\right\rangle_{h /(\gamma-N)}$, since for $x_{F} \rightarrow 1$ the latter has to vanish.

\section{2d. Comparison with Data}

In the parton model ${ }^{1)}$ the same parton fragmentation functions $D_{q}^{h}\left(z, p_{T}\right)$ appear both in $\mathrm{e}^{+} \mathrm{e}^{-}$annihilation and in lepto-production. Some support for this can be found in existing data. For example, in $\mu^{-} p \rightarrow \mu^{-}+h+X$ at FNAL ${ }^{8)}$ $\sigma_{\text {tot }} x_{F} \frac{d \sigma}{d x_{F}}$ can be approximately described by an exponential, $e^{-a x_{F}}$ with $\quad a \cong 3.4-4$. In $\mathrm{e}^{+} \mathrm{e}^{-}$annihilation, over the range $3 \leq \sqrt{Q^{2}} \leq 7.4 \mathrm{GeV}^{25}$ ), 
$Q^{2} \times \frac{d \sigma}{d x} \quad$ is roughly consistent with the same fall-off for $.3 \leqslant x \leqslant .8$. This rough agreement encourages us to proceed and discuss relations between $P_{T}$ distributions in the two types of processes. Estimating $\left\langle p_{T}^{2}\right\rangle_{h} / q$ from inclusive electroproduction in the parton fragmentation region we obtain a predicted curve for $\alpha(p)$ in $e^{+} e^{-}$annihilation.

In section 2c, we have found that the $P_{T}$ distribution of hadrons, produced in the current fragmentation region of leptoproduction, should broaden with their $X_{F}$. Such broadening has. indeed been found in previous electroproduction data ${ }^{9}$ ), and recently, in the FNAL muon-production data ${ }^{8)}$. The information available at present is not sufficient for checking the details of our prediction (namely, rise with $x_{F}^{2}$, see eq. (14)), but is sufficient for our purposes here. In the FNAL data ${ }^{8)}\left\langle p_{T}^{2}\right\rangle_{h /(\gamma-N)}$ obtains the value $.17-.21 \mathrm{GeV}^{2}$ for $.05 \leqslant x_{F} \leq .4$ and $.26-.42$ for $.4 \leq x_{F} \leqslant .85$. The two values quoted for each $x_{F}$ range arise from two different parametrizations of the data, and indicate errors involved. Using eq. (14) we see that the $.05 \leqslant x_{F} \leqslant .4$ region can give a reasonable estimate for $\left\langle p_{T}^{2}\right\rangle_{h / q}$ (since there $\left\langle x_{F}^{2}\right.$ ) is small). We choose $\left\langle p_{T}^{2}\right\rangle_{h / q}=.2$ as a representative value.

We now proceed to the calculation of $\alpha(p)$ in $e^{+} e^{-}$annihilation. In eq. (3) we set $\alpha_{j}=1$, and assume $f\left(x, p_{T}\right)=f(x) g\left(p_{T}\right)$. For $g\left(p_{T}\right)$ we try two forms: $e^{-B p_{T}^{2}}$ and $e^{-b p_{T}}$ (both with $\left\langle p_{T}^{2}\right\rangle_{h / q}=.2 \mathrm{GeV}^{2}$ ). The results are shown in Fig. 4, where the solid line corresponds to $e^{-B p_{T}^{2}}$ and the dashed line - to $e^{-b p_{T}}$. Notice that the model dependence is not more than a few percent for $p \geqslant 1 \mathrm{GeV}$. Moreover, the approximation $\alpha(p) \cong 1-2\left\langle p_{T}^{2}\right\rangle_{h / q} / p^{2}$ is also good to a few percent for $p \geqslant 1 \mathrm{GeV}$. The differences at low $p\left(p^{2} \cong\left\langle p_{T}^{2}\right\rangle_{h k_{q}}\right)$ are due to the different $p_{T}$ distributions. 
For the sake of completeness let us mention that for small $p$ eq. (3) gives

$$
\alpha\left(Q^{2}, p\right) \cong \int_{\alpha_{j}\left(Q^{2}\right) \cdot \frac{3 \sqrt{6} \pi}{32\left(\alpha_{j}+3\right)} \cdot \frac{p}{\sqrt{\left\langle p_{T}^{2}\right\rangle_{h / q}}}}\left(e^{\left.-b p_{T}\right)}\right.
$$

On Fig. 4 we have added the data for $\alpha$ at $\left.\sqrt{Q^{2}}=7.4 \mathrm{GeV} 5\right)$. The agreement is good but not very significant. After all, the model is not expected to apply to low momentum hadrons. And in the region where we expect the model to be valid $(p \geqslant 1 \mathrm{GeV})$ the errors in the data are large enough to exclude any clear cut conclusions.

\section{Remarks and Conclusions}

In this paper we have explored some consequences of the existence of jets, or of a transverse momentum cut-off in parton model language. We conclude with a few remarks.

1. If jets exist one can obtain the parameters $\alpha_{j}$ and $\left\langle p_{T}^{2}\right\rangle_{h / j e t}$ from inclusive experiments without having to find jets event-by-event. The same parameter $\left\langle p_{T}^{2}\right\rangle_{h / j e t}$ also appears in inclusive electroproduction in the current fragmentation region. 
2. The observation that $\alpha(p) \rightarrow 1$ for large $p$ in $e^{+} e^{-}$annihilation does not prove that jets exist. A strong two-particle correlation of the sort we have discussed (see section $2 \mathrm{~b}$.) with a bounded $\left\langle p_{T}^{2}\right\rangle_{2 / 1}$ is a definite hint that jets exist in the subset of events with at least one fast particle $\left(X_{1}>\sim .5\right.$, say). One should keep in mind that present data do not definitely prove the existence of jets.

3. The non-scaling effect of $\alpha(p) \neq 1$ is related to the deviation from scaling in $Q^{2} \frac{d \sigma}{d x}$, though in a model dependent fashion. This occurs even if (Feynman) scaling is assumed at the jet level. Thus this represents a sort of minimal scaling violation.

4. Under plausible simplifying assumptions $\alpha$ turns out to depend on the particle momentum $p$. It therefore seems useful to plot $\alpha$ versus $p$ for different $Q^{2}$ values rather than versus $x$.

5. The comparison between $e^{+} e^{-}$and electroproduction data should be viewed with caution, since $e^{+} e^{-}$physics exhibits an apparent threshold behaviour around $\sqrt{\mathrm{Q}^{2}} \cong 4 \mathrm{GeV}$, while there is as yet no evidence for such a dramatic behaviour in deep inelastic reactions.

6. The jet picture is unreliable for low momentum particles. Their distributions are probably determined by low energy hadron dynamics and not by the jet picture. Even in the latter $f\left(Q^{2}, x, P_{T}\right)$ is not expected to scale at very small $x$. Moreoever, for low momentum hadrons one has to include interference effects arising from an overlap of different jet axes in the production amplitude. For events with high momentum particles (i.e. a jet axis in each 
event) one can ignore this interference as we have done. The qualitative behaviour of the simple model is probably near the truth also for slow particles.

The parton model is not the only source of jet structure in $\mathrm{e}^{+} \mathrm{e}^{-}$annihilation. Resonance production models can also lead to jets. Models in which the photon decays into one light meson recoiling against a heavy resonance lead to a jet picture at intermediate $Q^{2}$. At high energies the jet either broadens or remains narrow depending on the average spin of the heavy resonance ${ }^{10)}$. Models in which the photon decays into two heavy states, which then decay similarly, can also have a two jet structure at intermediate energies. However, at high energies each jet should dissolve into sub-jets. The number of the latter increases with energy 11). In view of this one should keep an open mind about possible surprises at very high $Q^{2}$.

\section{Acknowledgement}

Two of us (M.G. and Y.Z.) would like to thank the DESY theory group for its hospitality; T.W. wishes to thank P. Zerwas for a discussion. 


\section{$\underline{\text { References }}$}

1. R.P. Feynman - Photon-Hadron Interactions (Benjamin, 1972).

2. F. Ravnda1 - Phys. Letters 43B (1973) 301.

3. S.D. Drel1, D.J. Levy and T.M. Yan, Phys. Rev. D1 (1970) 1617;

J.D. Bjorken and S.J. Brodsky, Phys. Rev. D1 (1970) 1416;

N. Cabibbo, G. Parisi and M. Testa, Nuovo Cim. Lett. 4 (1970) 1.

4. B. Richter - In Proceedings of the XVII International Conference on High Energy Physics (Rutherford Laboratory, 1974).

5. R.F. Schwitters - Talk presented in the 1975 International Symposium on Lepton and Photon Interactions at High Energies (Stanford, August, 1975).

6. J. Ellis - In Proceedings of the XVII International Conference on High Energy Physics (Rutherford Laboratory, 1974) and references therein.

7. R.C.E. Devenish, K. Koller, D. Schiller and T.F. Walsh (unpublished).

8. L. Mo - Talk presented in the 1975 International Symposium on Lepton and Photon Interactions at High Energies (Stanford, August, 1975).

9. F.W. Brasse - In Proceedings of the 6th International Symposium on Electron and Photon Interactions at High Energies (Bonn, 1973).

10. N.S. Craigie and K.W. Rothe - Nuc1. Phys. B82 (1974) 370, 381; N.S. Craigie and T.F. Walsh - Annals of Physics (in print).

11. A.M. Polyakov - JETP 32 (1971) 296; JETP 33 (1971) 850. 


\section{Figure Captions}

Fig. 1 Geometry of $\mathrm{e}^{+} \mathrm{e}^{-} \rightarrow h+X$ in jets.

Fig. 2 Relative $p_{T^{-}}$geometry in $e^{+} e^{-} \rightarrow h_{1}+h_{2}+X$

Fig. $3 P_{T}$ geometry for parton fragmentation region in leptoproduction
a) before interaction
b) after interaction.

$\underline{\text { Fig. } 4}$ Plot of $\alpha(p)$ for $\mathrm{e}^{+} \mathrm{e}^{-}$annihilation. Predictions: solid line $-e^{-B p_{T}^{2}}$; dashed line $-e^{-b p_{T}}$. Data taken from ref. 5). 


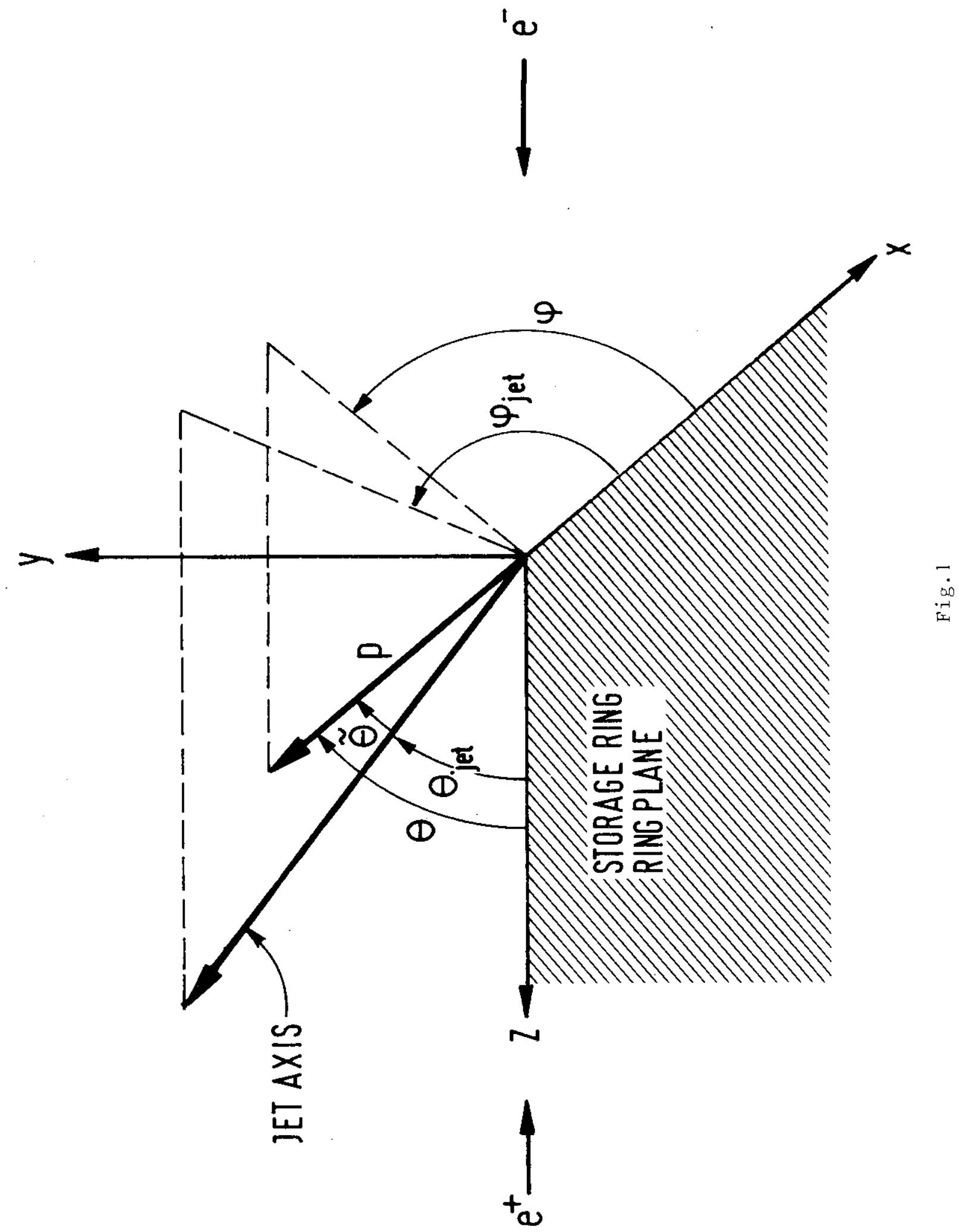




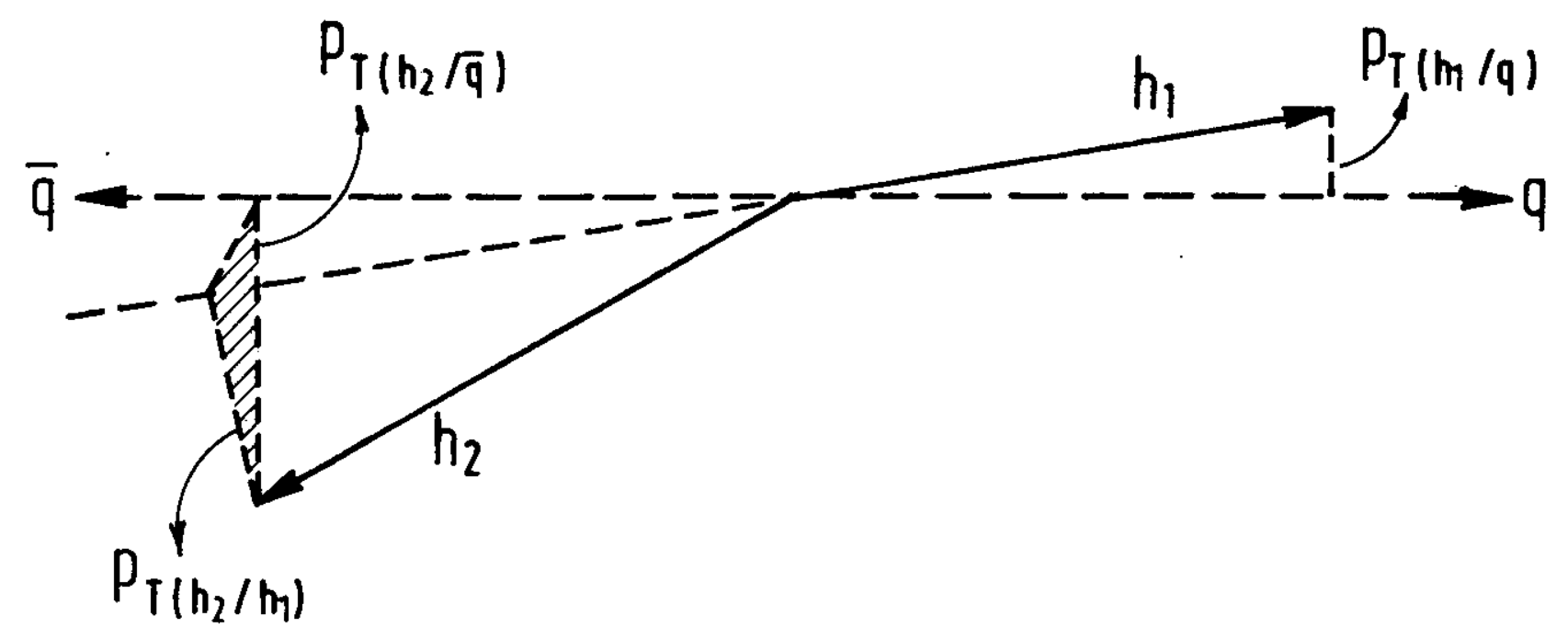

Fig. 2 

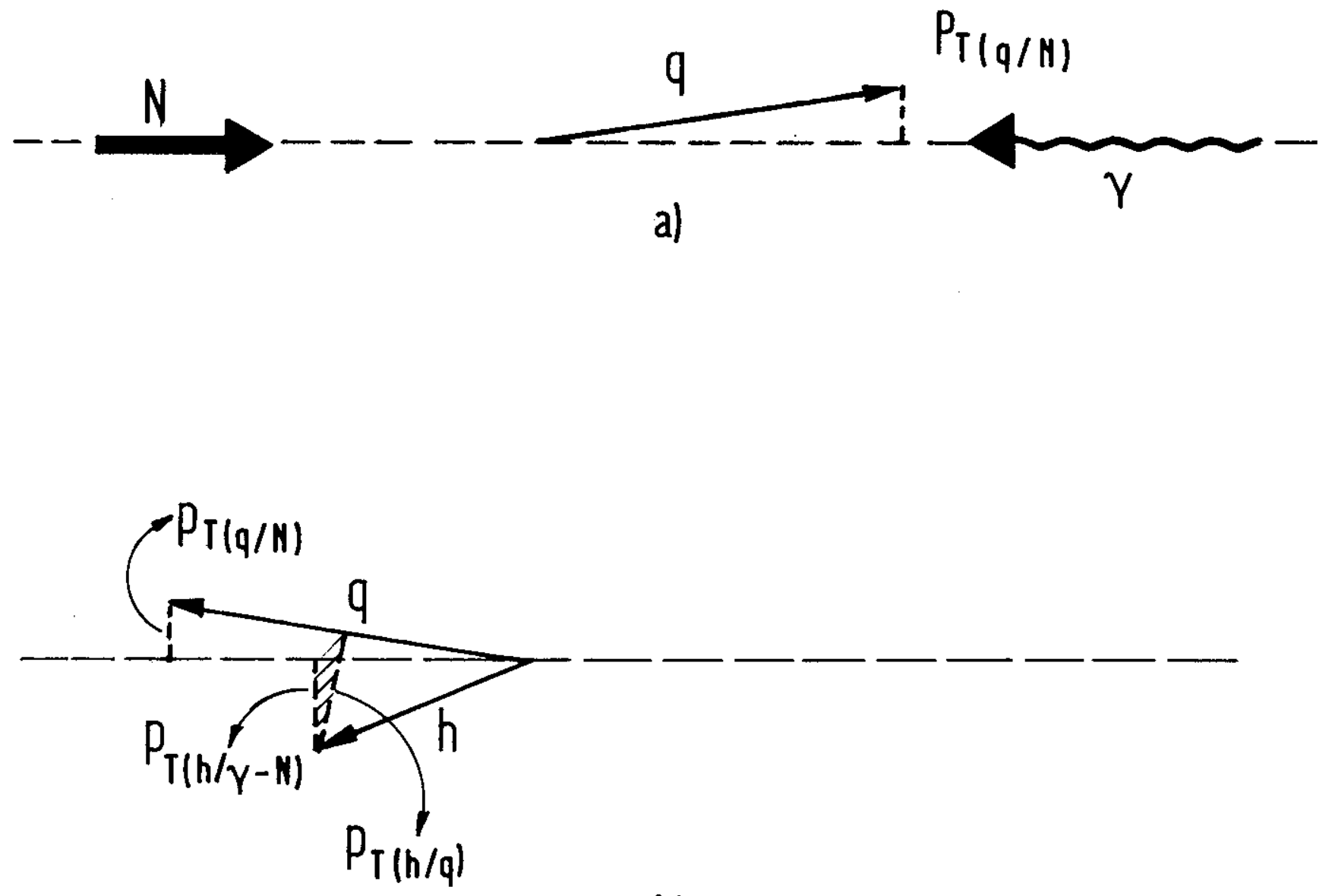

b)

Fig. 3 


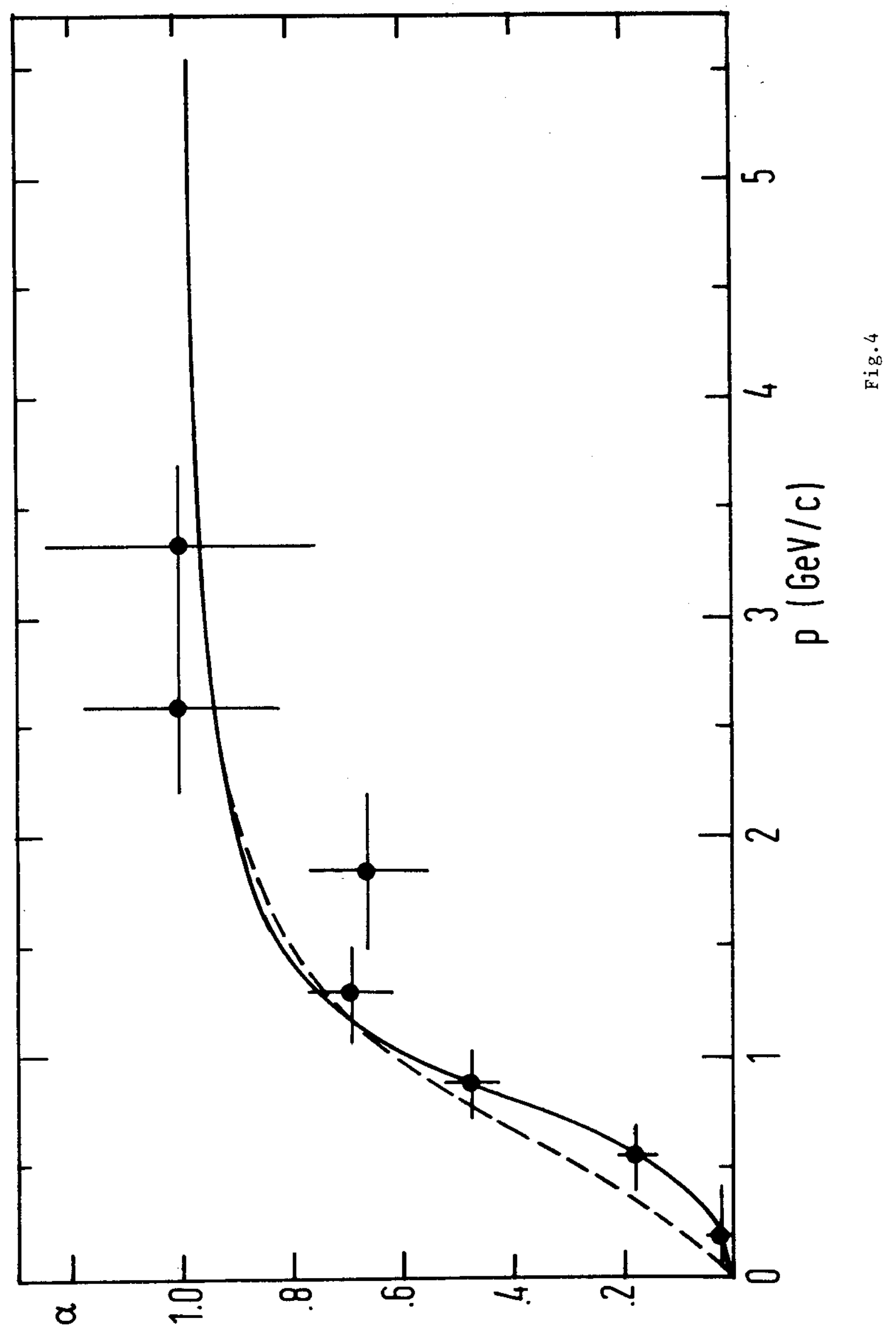

